\title{
Fatores dietéticos na prevenção e tratamento de comorbidades associadas à síndrome metabólica
}

\author{
Dietary factors in preventing and treating comorbidities \\ associated with the metabolic syndrome
}

Cláudia Roberta Bocca SANTOS ${ }^{1}$

Emilson Souza PORTELLA²

Sonia Silva AVILA²

Eliane de Abreu SOARES 2,3

\section{R E S U M O}

A síndrome metabólica constitui um fator de risco cardiovascular, sendo caracterizada pela associação de hipertensão arterial sistêmica, obesidade abdominal, tolerância à glicose prejudicada, hipertrigliceridemia e baixas concentrações sangüíneas de HDL-colesterol, além dos estados pró-trombótico e pró-inflamatório observados. No entanto, a alimentação adequada, associada a outras modificações no estilo de vida, tais como prática regular de atividade física e abandono do tabagismo, contribui para um melhor controle da doença, prevenindo suas complicações e aumentando a qualidade de vida. Desse modo, o objetivo deste estudo foi revisar, na literatura científica, o papel da dieta na prevenção e tratamento da síndrome metabólica. Os trabalhos sobre o assunto informam que a terapia nutricional visa a limitar o consumo de gorduras saturadas e ácidos graxos trans isômeros, os principais envolvidos no aumento da colesterolemia. O aporte protéico deve ser semelhante às recomendações da população geral, com ênfase no consumo de proteínas de origem vegetal e de peixe. Outro fator importante refere-se ao índice glicêmico dos alimentos: dietas de alto índice glicêmico estão relacionadas à promoção da resistência insulínica, obesidade e diabetes mellitus tipo 2 . Quanto às fibras alimentares, muitos estudos comprovam que a dieta rica em fibras diminui o risco de doenças coronarianas e diabetes mellitus tipo 2, além de contribuir para melhor controle glicêmico. Portanto, a alimentação adequada constitui um fator indispensável não somente no tratamento, como também na prevenção da síndrome metabólica.

Termos de indexação: diabetes mellitus; dieta; doenças cardiovasculares; nutrição; síndrome metabólica.

\footnotetext{
1 Iniciação Científica, Instituto de Nutrição, Universidade do Estado do Rio de Janeiro. Rio de Janeiro, RJ, Brasil.

2 Departamento de Nutrição Básica e Experimental, Instituto de Nutrição, Universidade do Estado do Rio de Janeiro. Rua São Francisco Xavier, 524, Pavilhão João Lyra Filho, Bloco D, $12^{\circ}$ andar, Maracanã, 20650-013, Rio de Janeiro, RJ, Brasil. Correspondência para/Correspondence to: E.A. SOARES.

3 Centro de Ciências da Saúde, Universidade Federal do Rio de Janeiro. Rio de Janeiro, RJ, Brasil.
} 


\section{A B S T R A C T}

The metabolic syndrome constitutes a cardiovascular risk factor characterized by the association of high blood pressure, abdominal obesity, impaired glucose tolerance, high plasma triglycerides and low HDL-cholesterol concentration, beyond the observed prothrombotic and proinflammatory states. Otherwise, an adequate diet associated with other lifestyle modifications, such as regular physical activity and curb smoking contribute to an improved control of the disease, preventing its complications and increasing the quality of life. In this way, the proposal of this study was to review, in the scientific literature, the role of the diet in preventing and treating the metabolic syndrome. The articles on this matter inform that the nutritional therapy aims to limit the consumption of saturated fats and trans isomeric fatty acids, the main components involved in increasing cholesterolemia. The quantity of protein must be similar to the recommendations for the general population, with emphasis on plant and fish protein. Another important issue is the glycemic index of foods: diets with high glycemic index promote insulin resistance, obesity and type 2 diabetes mellitus. In relation to dietary fibers, many studies show that a diet rich in fibers reduces the risk of coronary diseases and type 2 diabetes mellitus, and also contributes to improve glycemic control. Therefore, an adequate diet not only constitutes an indispensable factor in the treatment but also in the prevention of the metabolic syndrome.

Indexing terms: diabetes mellitus; diet; cardiovascular diseases; nutrition; metabolic syndrome.

\section{N T R O D U Ç Ã O}

A crescente incidência da síndrome metabólica nos Estados Unidos deve-se ao maior número de casos de obesidade e de diabetes mellitus tipo 2 nas últimas três décadas, afetando não somente a população adulta, como também crianças e adolescentes. Nestes, a síndrome metabólica é responsável pela promoção de aterosclerose prematura e por riscos precoces da doença cardiovascular ${ }^{1}$. Nesse sentido, sabe-se da importância da alimentação na prevenção e tratamento de cardiopatias. No entanto, o efeito da composição da dieta ainda não está completamente estabelecido. Por muitos anos, dietas hipolipídicas foram recomendadas, a fim de prevenir e tratar as doenças cardiovasculares. Hoje, tais recomendações são discutíveis, em função do atual conhecimento de que dietas hiperglicídicas podem promover a síndrome metabólica. Além disso, discute-se a relação entre síndrome metabólica e fibras alimentares, índice glicêmico e gorduras monoinsaturadas. Neste contexto, o objetivo deste trabalho foi avaliar o papel da alimentação na prevenção, como também no tratamento da síndrome metabólica. Para levantamento bibliográfico, foram utilizados como base de dados Pubmed, Medline, Periódicos Capes, ScienceDirect e MDConsult, sendo escolhidas como palavras-chave síndrome metabólica, dieta, nutrição e doença cardiovascular. Foram utilizadas referências dos últimos cinco anos.

A síndrome metabólica, já conhecida como síndrome da resistência à insulina, síndrome $X$, síndrome plurimetabólica ou quarteto mortal, corresponde a um distúrbio metabólico complexo ${ }^{1-3}$, caracterizado, de acordo com a World Health Organization $(\mathrm{WHO})^{4}$, pela associação de tolerância à glicose prejudicada/diabetes mellitus e/ou resistência insulínica, além de dois ou mais dos seguintes fatores: hipertensão arterial sistêmica (valores superiores a 140/90mmHg); hipertrigliceridemia (concentrações plasmáticas maiores que $150 \mathrm{mg} / \mathrm{dl}$ ) e/ou concentrações séricas de lipoproteína de alta densidade (HDL-c) inferiores a $35 \mathrm{mg} / \mathrm{dl}$ e $39 \mathrm{mg} / \mathrm{dl}$, em homens e mulheres, respectivamente; obesidade central (definida por relação cintura-quadril superior a 0,90 para o sexo masculino e 0,85 para o feminino) e/ou Índice de Massa Corporal (definido como o resultado da divisão da massa corporal em kilogramas pela estatura em metros ao quadrado) maior que $30 \mathrm{~kg} / \mathrm{m}^{2}$; microalbuminúria (taxa de excreção urinária de albumina maior ou igual a $20 \mu \mathrm{g} / \mathrm{min}$ ou razão albumina: creatinina maior ou igual a $30 \mathrm{~g} / \mathrm{mg}$ ).

Recentemente, o National Cholesterol Education Program (NCEP) ${ }^{5}$ norte-americano 
descreveu que o diagnóstico de síndrome metabólica é determinado pela presença de três ou mais das seguintes afecções: obesidade abdominal (determinada por valores de circunferência de cintura superiores a $102 \mathrm{~cm}$ e $88 \mathrm{~cm}$, em homens e mulheres, respectivamente), hipertensão arterial sistêmica (definida por níveis pressóricos iguais ou maiores que $130 / 85 \mathrm{mmHg}$ ), tolerância à glicose prejudicada (glicemia de jejum entre 110 e $125 \mathrm{mg} / \mathrm{dl}$ ), hipertrigliceridemia (valores iguais ou superiores a $150 \mathrm{mg} / \mathrm{dl}$ ) e baixas concentrações sangüíneas de HDL-c (inferiores a 40mg/dl para homens e $50 \mathrm{mg} / \mathrm{dl}$ para mulheres).

Segundo Lorenzo et al. ${ }^{6}$, a definição NCEP 5 detecta maior número de indivíduos em risco de diabetes $(48,7 \%)$ que a definição da $\mathrm{WHO}^{4}$ $(41,3 \%)$, uma vez que a síndrome metabólica constitui um fator preditivo de desenvolvimento de diabetes mellitus, independentemente de outros fatores de risco.

A fim de comparar a prevalência de síndrome metabólica utilizando essas duas definições, Ford \& Giles ${ }^{7}$ avaliaram 8608 participantes com mais de 20 anos de idade, do National Health and Nutrition Examination Survey III (NHANES III), encontrando diferentes prevalências de acordo com o critério utilizado. Segundo a $\mathrm{WHO}^{4}, \mathrm{a}$ prevalência identificada nesse estudo foi de $25,1 \%$, enquanto que pelo critério do NCEP ${ }^{5}$, foi $23,9 \%$. Além disso, $86,2 \%$ dos indivíduos avaliados foram classificados como sendo ou não portadores da síndrome metabólica, dependendo do parâmetro usado. De acordo com esse estudo, pode-se observar a necessidade de estabelecer uma definição universalmente aceita de síndrome metabólica.

\section{Prevalência}

A síndrome metabólica vem se tornando cada vez mais comum nos Estados Unidos, sendo considerada um fator tão importante quanto o tabagismo no desenvolvimento de doenças cardiovasculares prematuras 5 .

A fim de estimar a prevalência de síndrome metabólica em adultos norte-americanos, Ford et al. ${ }^{8}$ analisaram, num estudo transversal com dados do NHANES III, 8814 homens e mulheres com idade igual ou superior a 20 anos. Em adultos com idade entre 20 e 29 anos, a prevalência, segundo critério do NCEP5, foi igual a 6,7\%, aumentando para $43,5 \%$ para participantes com idade entre 60 e 69 anos e 42,0\% para aqueles com, no mínimo, 70 anos. Atualmente, pelo menos 47 milhões de americanos são portadores de síndrome metabólica ${ }^{8,9}$.

Isomaa et al. ${ }^{2}$ avaliaram 4483 indivíduos, com idade entre 35 e 70 anos, da Finlândia e Suécia. Destes, 1697 eram diabéticos tipo 2, 798 possuíam glicemia de jejum alterada (IFG) e 1988 eram resistentes à insulina com tolerância à glicose normal (NGT). A síndrome metabólica esteve presente em $10 \%$ dos indivíduos com NGT, 50\% daqueles com IFG e $80 \%$ dos diabéticos tipo 2, segundo definição da $\mathrm{WHO}^{4}$.

Tendo como base a definição do NCEP5, Santos et al. ${ }^{10}$ realizaram um estudo transversal com 38 diabéticos tipo 2, 18 homens e 20 mulheres, atendidos em um hospital universitário do Rio de Janeiro, virgens de tratamento dietoterápico. Nele, foi detectado que $89 \%$ da parcela masculina e $95 \%$ da feminina eram portadores da síndrome metabólica.

\section{FATORES DE RISCO}

A genética, o sedentarismo, o tabagismo, o ganho ponderal progressivo e uma dieta rica em carboidratos refinados, gorduras saturadas e pobre em fibras alimentares contribuem para o desenvolvimento da síndrome metabólica, considerada um fator que promove a aterosclerose e eleva o risco cardiovascular ${ }^{9}$.

Resistência insulínica: a resistência à insulina ocorre quando uma concentração normal desse hormônio produz uma menor resposta biológica nos tecidos periféricos, como músculo, fígado e tecido adiposo ${ }^{11}$. A ativação do receptor de insulina resulta na translocação da proteína transportadora de glicose 4 (GLUT4) do citosol para 
a membrana celular, o que permite a entrada de glicose na célula. A resistência insulínica pode decorrer de diversos fatores: defeitos na secreção e/ou ação da insulina por menor número de receptores ou menor afinidade desses, redução na quantidade de GLUT4 ou na translocação de GLUT4 para a membrana, sendo este último considerado como o fator mais importante ${ }^{12,13}$.

O excesso de gordura corporal (em especial a obesidade abdominal), o sedentarismo e a predisposição genética podem promover a resistência à insulina, que está intimamente relacionada à síndrome metabólica, porém os mecanismos para tal associação não estão bem esclarecidos $^{5}$. Sabe-se que, em resposta à resistência tecidual, a secreção de insulina é aumentada, resultando em hiperinsulinemia ${ }^{12}$. A resistência insulínica é observada anos antes do diagnóstico de diabetes mellitus tipo $2^{14}$.

Vários métodos são utilizados a fim de mensurar a resistência insulínica. Entre eles, o Homeostasis Assessment Model (HOMA), um modelo matemático, que permite obter valores para sensibilidade à insulina e função da célula $\beta$, se a glicemia de jejum e a concentração de insulina/peptídeo C plasmática em jejum são conhecidas ${ }^{11}$.

Entre outras anormalidades associadas à resistência insulínica e à hiperinsulinemia, incluem-se aumento da aderência mononuclear, diminuição da vasodilatação endotélio-dependente $^{15}$, elevadas concentrações séricas de ácido úrico e microalbuminúria. Pode-se observar, como achados clínicos, esteatose hepática, ovário policístico e acanthosis nigricans ${ }^{3}$.

Hipertensão arterial sistêmica: a hiperinsulinemia poderia elevar a pressão arterial por intermédio da ativação do sistema nervoso simpático, do comprometimento da vasodilatação periférica, da maior resposta a angiotensina e do aumento da reabsorção renal de sódio e água, com conseqüente sobrecarga de volume ${ }^{12,14,16}$. Santos et al. ${ }^{10}$ verificaram que $84 \%$ da amostra de diabéticos, de ambos os sexos, apresentavam níveis pressóricos elevados.
Dislipidemias: a forma mais comum de dislipidemia associada à síndrome metabólica, chamada dislipidemia aterogênica, é caracterizada por três anormalidades lipídicas: hipertrigliceridemia, baixas concentrações plasmáticas de HDL-c e partículas de lipoproteína de baixa densidade ( $L D L-C$ ) pequenas e densas. A sua etiologia está relacionada à resistência insulínica, na qual, em virtude do menor metabolismo de lipoproteína de muito baixa densidade (VLDL-c), decorrente da hiperinsulinemia, a concentração plasmática de triglicerídeos encontra-se aumentada, enquanto a de HDL-c está diminuída ${ }^{12,17}$. A hipertrigliceridemia é também causada pela maior síntese de apolipoproteína C-III, que interfere na ação da lipoproteína lipase, responsável pela hidrólise dos triglicerídeos da partícula de VLDL-c. Além disso, a apolipoproteína C-III interfere na captação de remanescentes de VLDL-c pelos receptores de LDL-c nas células hepáticas. Tais mecanismos levam ao acúmulo de triglicerídeos na corrente sanguínea ${ }^{18}$.

A concentração de LDL-c na resistência insulínica é discretamente aumentada ou normal, porém a eletroforese revela a presença de partículas pequenas e densas ${ }^{12,14,17}$. A resistência insulínica provoca maior oxidação dessas, pois as lipoproteínas glicosiladas são mais suscetíveis à oxidação, aumentando a aterogenicidade ${ }^{12}$.

Alguns estudos realizados demonstram uma elevada prevalência de dislipidemias em portadores de síndrome metabólica. Santos e colaboradores ${ }^{10}$ observaram que $87 \%$ dos diabéticos possuíam uma ou mais anormalidades lipídicas (hipertrigliceridemia, hipercolesterolemia e/ou baixas concentrações sanguíneas de HDL-C), sendo que desses $100 \%$ apresentavam sobrepeso ou obesidade. Com o objetivo de determinar a prevalência de diabetes mellitus e seus fatores de risco em adultos, Souza et al. ${ }^{19}$ observaram que excesso de peso, hipertensão arterial sistêmica e dislipidemias (hipertrigliceridemia, hipercolesterolemia e baixa concentração plasmática de $\mathrm{HDL}-\mathrm{c}$ ) foram mais prevalentes em diabéticos do que em não diabéticos, sendo as diferenças estatisticamente significantes. 
Obesidade abdominal: a maioria dos casos de síndrome metabólica ocorre em indivíduos com excesso de peso $^{3}$, o que, por si só, prejudica a sensibilidade à insulina, que diminui em até $40 \%$ quando o indivíduo apresenta massa corporal maior que $35 \%$ a $40 \%$ da ideal ${ }^{14}$. Santos et al. ${ }^{10}$ verificaram que apenas $8 \%$ dos 49 diabéticos estudados possuíam Índice de Massa Corporal (IMC) de acordo com os padrões de normalidade. O excesso de gordura corporal leva ao acúmulo de lipídeos nos tecidos, notavelmente no tecido adiposo, músculos, fígado e células $\beta$ pancreáticas, o que parece induzir as alterações bioquímicas ocorridas na síndrome metabólica ${ }^{3}$.

Mesmo pacientes não obesos podem apresentar aumento da gordura corporal predominantemente na região abdominal ${ }^{13}$. Em 84\% dos pacientes atendidos em um hospital universitário da cidade do Rio de Janeiro ${ }^{10}$, a circunferência abdominal era superior aos limites estabelecidos pelo NCEP5. Outros estudos têm demonstrado a correlação existente entre a obesidade abdominal e os demais componentes da síndrome metabólica ${ }^{3,5,17}$. Depósitos viscerais de gordura possuem turnover mais acelerado que em outras regiões, elevando as concentrações do inibidor do ativador de plasminogênio-1 (PAI-1), citocinas inflamatórias e ácidos graxos não-esterificados (NEFA) no sistema porta. A maior liberação de NEFA do tecido adiposo estimula a gliconeogênese, inibe a depuração hepática de insulina e provoca acúmulo de triglicerídeos no fígado e músculo, resultando em hiperglicemia e conseqüente hiperinsulinemia. Assim, o acúmulo de gordura no músculo leva à resistência insulínica, enquanto no fígado, promove a dislipidemia aterogênica $a^{3,17,20}$.

Em obesos, as concentrações séricas de angiotensinogênio estão aumentadas, devido à sua maior síntese pelos adipócitos, gerando mais angiotensina II e conseqüente elevação da pressão arterial. Desse modo, a angiotensina II atua como elo entre a obesidade e a hipertensão arterial ${ }^{16}$.

Vários hormônios possuem papel fundamental na manutenção do peso corpóreo. A leptina é um peptídeo secretado pelos adipócitos, que parece ser importante na regulação da quantidade de gordura corporal. A concentração de leptina é proporcional ao número e tamanho dos adipócitos. No entanto, como há variação nas concentrações de leptina, mesmo entre indivíduos com semelhante composição corporal, acreditase que existam outros fatores que influenciem as concentrações plasmáticas de leptina ${ }^{21}$. Um estudo foi publicado com o objetivo de examinar se as modificações de estilo de vida afetariam as concentrações plasmáticas de leptina. Participaram da pesquisa 186 homens com síndrome metabólica e sedentários, que foram divididos em quatro grupos: um grupo controle, um grupo sob intervenção dietética, um submetido a programa de exercícios físicos e o quarto grupo combinando dieta hipocalórica e exercícios. Dados demográficos, de ingestão dietética e de nível de atividade física foram coletados, e foram mensuradas as concentrações plasmáticas de leptina antes e após o período de intervenção de um ano. As concentrações de leptina, o IMC e a gordura corporal diminuíram, em associação com a redução da ingestão dietética e o aumento de atividade física. As modificações de estilo de vida a longo prazo, com a diminuição da ingestão de lipídeos e o aumento de atividade física, reduziram as concentrações plasmáticas de leptina. Essa alteração reflete, possivelmente, um efeito direto da dieta e do exercício físico sobre as concentrações plasmáticas de leptina ${ }^{21}$. A hiperinsulinemia e a hiperleptinemia configuram o perfil da maior parte dos obesos ${ }^{16}$.

Estados pró-trombótico e pró-inflamatório: a maior liberação de PAI-1 e citocinas inflamatórias é responsável pelos estados pró-trombótico e pró-inflamatório, respectivamente ${ }^{3}$. O aumento das concentrações de PAI-1 promove menor ação fibrinolítica. Além disso, há maior produção de trombina e fibrina, em função da ativação das células endoteliais, aumento da agregação plaquetária, ativação do fator de coagulação VII e elevadas concentrações dos fatores X, IX e da protrombina ${ }^{18}$. 
O aumento das concentrações plasmáticas de interleucina-6 (IL-6) e da proteína C reativa $(P C R)$ está associado à resistência insulínica, ao diabetes mellitus tipo 2 e a eventos cardiovasculares, tanto em indivíduos aparentemente saudáveis quanto naqueles em alto risco ${ }^{22}$. A PCR é um marcador inflamatório do organismo e, em concentrações elevadas, corresponde a um fator de risco cardiovascular ${ }^{23}$, estando correlacionada à obesidade, resistência insulínica e hiperglicemia. Tal associação sugere que o diabetes mellitus tipo 2 e a resistência à insulina podem ser conseqüência de resposta de fase aguda, refletindo uma adaptação crônica do sistema imune ${ }^{24}$. Han et al. ${ }^{23}$, visando a avaliar se as concentrações de PCR representavam fator de risco de desenvolvimento de diabetes mellitus e síndrome metabólica, estudaram 729 mulheres e 515 homens mexicanos durante seis anos. Nesse estudo, a concentração de PCR não constituiu fator preditivo de desenvolvimento de síndrome metabólica em homens, apenas em mulheres.

Sabe-se que o fator de necrose tumoral alfa ( $\alpha$-TNF) está envolvido na gênese da resistência insulínica, por inibir a fosforilação de seus receptores. Em obesos, os níveis de $\alpha$-TNF estão aumentados. Esta citocina aumenta a produção de endotelina 1 e de angiotensinogênio, refletindo a disfunção endotelial que pode ocorrer em obesos hipertensos ${ }^{16}$, além de estimular a lipólise e inibir a lipogênese ${ }^{14}$. O $\alpha$-TNF associa-se, ainda, à promoção da aterosclerose por alterar a estabilidade plaquetária, a permeabilidade do endotélio e ativar monócitos e macrófagos ${ }^{25}$.

A homocisteína, em altas concentrações, possui efeitos citotóxicos ao endotélio vascular, por acelerar a produção de espécies reativas de oxigênio, estimular a ativação da cascata de coagulação, aumentar a adesividade plaquetária, prejudicar a vasodilatação dependente de óxido nítrico e estimular a oxidação de LDL-c ${ }^{26,27}$. Além disso, pode induzir à resistência insulínica, levando à hiperinsulinemia compensatória, o que poderia alterar ainda mais o metabolismo de homocisteína, que se acumularia no plasma ${ }^{26}$. Portanto, as concentrações de homocisteína podem aumentar na resistência insulínica. Em diabéticos tipo 2, a hiperhomocisteinemia correlaciona-se ainda à microalbuminúria ${ }^{27}$.

Meigs et al. ${ }^{26}$ buscaram avaliar a relação entre as concentrações de homocisteína e a síndrome metabólica. Foram avaliadas as concentrações em jejum de homocisteína, folato, vitaminas do complexo $B$, creatinina, concentrações de insulina em jejum e de $2 \mathrm{~h}$ e glicemia após teste oral de tolerância à glicose $(75 \mathrm{~g})$ de 2214 indivíduos participantes do Framingham Offspring Study. Meigs et al. ${ }^{26}$ concluíram que a hiperhomocisteinemia e a excreção anormal de albumina na urina estão associadas à hiperinsulinemia. Uma limitação do estudo foi a carência de um método específico para mensuração da resistência insulínica, o que enfraqueceria as associações encontradas e subestimaria o efeito da resistência à insulina sobre as concentrações de homocisteína ${ }^{26}$.

Cada uma das afecções descritas concorre isoladamente para promoção da aterosclerose, no entanto, a combinação desses fatores mostra-se muito mais aterogênica, elevando o risco de morbimortalidade cardiovascular, independentemente da concentração sérica de LDL-C $C^{4,5}$.

Para avaliar o efeito sobre a reatividade vascular, Hamdy et al. ${ }^{28}$ estudaram 24 indivíduos obesos com síndrome metabólica, após seis meses de perda ponderal e exercício físico de intensidade moderada. Os resultados indicaram perda ponderal de aproximadamente $6,6 \%$, com melhora na sensibilidade à insulina e na função endotelial, redução de marcadores de ativação endotelial e de coagulação, a despeito do grau de tolerância à glicose. No entanto, vale ressaltar que o estudo não contou com um grupo controle de homens sadios e eutróficos. Além disso, não foi determinado, separadamente, o efeito da perda ponderal e do exercício físico sobre os resultados encontrados. Outra pesquisa, na qual foi observada perda ponderal de $14 \mathrm{~kg}$ em 24 semanas, de 36 indivíduos obesos, na sua maioria mulheres, em dieta hipocalórica, verificou melhoria dos fatores 
hemostáticos, com redução em $12,0 \%$ do fator de coagulação VII, em $6,0 \%$ de fibrinogênio e um declínio de 34,0\% no PAI-1, quando comparados aos valores iniciais do estudo ${ }^{29}$.

\section{A L I M E N T A Ç Ã O}

A terapia nutricional com os portadores de síndrome metabólica deve focar não somente o controle glicêmico, como também reduzir os demais fatores de risco cardiovasculares ${ }^{4}$. Dessa forma, a estratégia inicial para o tratamento desta síndrome baseia-se na modificação de suas causas originais: excesso de peso e sedentarismo, visando à diminuição da resistência insulínica ${ }^{5}$. As mudanças no estilo de vida, com perda ponderal moderada, porém progressiva, são a conduta aceita como mais efetiva. Além disso, perda de peso, com aumento da atividade física, reduz em $60 \%$ a probabilidade da tolerância à glicose prejudicada evoluir para o diabetes mellitus ${ }^{3}$.

O tratamento dietoterápico de pacientes com síndrome metabólica deve priorizar a perda ponderal, o que, por si só, melhora a sensibilidade à insulina e confere benefícios adicionais em relação às demais anormalidades características da síndrome ${ }^{28-31}$. A perda de $5 \%$ a $10 \%$ da massa corporal é suficiente para conferir efeito benéfico clínico, sendo que este é preservado desde que não haja ganho de peso ${ }^{30}$.

Lipídeos: a exposição crônica à glicose e aos ácidos graxos livres pode inibir a secreção insulínica. O efeito dos ácidos graxos é variável, elevando a secreção insulínica com o aumento da cadeia e diminuindo com o grau de insaturação. Assim, ácidos graxos de cadeia longa potencializam a secreção de insulina, em resposta à concentração basal de glicose ${ }^{32}$.

Recentemente, El-Assaad et al. ${ }^{33}$ buscaram avaliar o efeito in vitro de diferentes ácidos graxos livres sobre as células $\beta$ pancreáticas, na presença ou ausência de elevadas concentrações de glicose. Segundo tais autores, nos diabéticos tipo 2 haveria menor número de células $\beta$, devido ao maior estímulo à apoptose, que pode ser induzida por elevadas concentrações de ácidos graxos livres e de glicose. Nesse estudo, foi encontrado que os ácidos graxos livres teriam diferentes efeitos sobre essas células em concentrações elevadas de glicose: os ácidos palmítico e esteárico (saturados) demonstraram maior toxicicidade, o linoléico (poliinsaturado) demonstrou toxicidade moderada e o oléico (monoinsaturado) demonstrou pouco ou nenhum efeito tóxico.

O objetivo primário da terapia nutricional é limitar a ingestão de gorduras saturadas, por constituírem o principal fator determinante da elevação das concentrações plasmáticas de LDL-C ${ }^{13}$. Em seguida, os lipídeos que mais contribuem para esse aumento são os ácidos graxos trans isômeros e, em menor grau, o colesterol dietético ${ }^{34}$.

As gorduras saturadas inibem a depuração plasmática de LDL-C, além de permitir maior entrada de colesterol nessas partículas ${ }^{34}$. A ingestão excessiva desse tipo de gordura está associada à alteração na ação da insulina, com risco de prejuízo à tolerância à glicose e de elevação da glicemia de jejum ${ }^{30,35}$.

Os ácidos graxos trans isômeros são produzidos durante o processo de hidrogenação dos óleos vegetais ${ }^{34,36}$. A elevada ingestão de ácidos graxos trans aumenta o risco de doença cardiovascular e diabetes mellitus, porém os mecanismos envolvidos ainda não estão bem elucidados. Sabe-se que modificam o perfil lipídico, diminuindo as concentrações de HDL-c e aumentando as de LDL-c e lipoproteína(a) 13,25,34. O consumo médio de ácidos graxos trans, em países industrializados, é em torno de 4\% a 7\% da ingestão total de lipídeos ${ }^{25}$, sendo que a atual recomendação da $\mathrm{WHO}^{35}$ é de menos de $1 \%$ do Valor Energético Total (VET).

A fim de avaliar a relação entre o consumo de ácidos graxos trans isômeros e marcadores inflamatórios, como PCR, IL-6 e os receptores 1 e 2 do $\alpha$-TNF, Mozaffarian et al. ${ }^{25}$ avaliaram 823 enfermeiras que participaram de dois estudos epidemiológicos americanos, Nurses' Health Study 
e Nurses' Health Study II. Foram mensuradas as concentrações dos referidos marcadores inflamatórios, e dois questionários de freqüência de consumo alimentar semi-quantitativos foram utilizados, a fim de estimar a ingestão dietética usual. O consumo de ácidos graxos trans isômeros foi positivamente associado com os receptores 1 e 2 do a-TNF. Tal associação não se alterou significativamente, quando ajustada para IMC, tabagismo, nível de atividade física, uso de aspirina ou droga antiinflamatória não esteróide, consumo de álcool e ingestão de gordura saturada, proteína, ácidos graxos ômega 3 e 6, fibras alimentares e energia. Os autores encontraram associação entre os ácidos graxos trans, PCR e IL- 6 apenas em mulheres com IMC aumentado. No entanto, em função de estimativas errôneas do conteúdo de ácidos graxos trans isômeros de alguns alimentos, pode ter havido erro ao quantificar a ingestão desses. Além disso, segundo os próprios autores, a alteração da dieta usual, no dia da coleta de sangue, pode ter afetado a resposta inflamatória.

Quanto ao colesterol dietético, possui menor efeito na colesterolemia que as gorduras saturadas $^{34,36}$. Segundo o NCEP5, sua ingestão deve ser inferior a 200mg diários. Atualmente, a $\mathrm{WHO}^{35}$ preconiza que o consumo de colesterol deve ser inferior a $300 \mathrm{mg} / \mathrm{dia}$. Já os fitoesteróis reduzem as concentrações de LDL-cem $6 \%$ a $15 \%$, quando a quantidade diária consumida é de 2 a $3 \mathrm{~g}$, com pouco ou nenhum efeito nas concentrações de HDL-c e triglicerídeos 5 .

Os ácidos graxos ômega-6, em elevadas quantidades, podem provocar pequenas reduções nas concentrações séricas de HDL-c e triglicerídeos $^{5}$, além de apresentarem maior suscetibilidade à oxidação ${ }^{34}$. Já os ácidos graxos ômega-3 podem diminuir as concentrações de triglicerídeos (efeito secundário à redução da síntese de VLDL-c). Diminuem, ainda, a adesividade plaquetária e promovem pequena redução na pressão arterial $^{5,34}$. Os ácidos graxos parecem também modular a liberação de diferentes citocinas, Fernández-Real et al. ${ }^{22}$ encontraram forte associação entre os ácidos graxos dietéticos e a atividade inflamatória. O percentual de ácidos graxos ômega- 6 foi positivamente associado à concentração circulante de IL-6, enquanto que os ácidos graxos ômega-3 possuíam associação negativa com as concentrações sanguíneas de PCR em indivíduos com excesso de peso.

As gorduras monoinsaturadas fortalecem as partículas de LDL-C, tornando-as menos propensas à oxidação. Em substituição às gorduras saturadas, promovem efeitos benéficos nas concentrações de colesterol total e de HDL- $\mathrm{c}^{34}$.

Samaha et al. ${ }^{37}$, após o uso de dietas hipoglicídicas e hiperlipídicas por um período de seis meses, observaram melhora na sensibilidade à insulina e nas concentrações plasmáticas de triglicerídeos em 132 indivíduos obesos com IMC em torno de $43 \mathrm{~kg} / \mathrm{m}^{2}$. Foram identificadas elevadas prevalências de diabetes mellitus e síndrome metabólica (39\% e 43\%, respectivamente).

Alguns estudos têm demonstrado efeitos benéficos da chamada dieta mediterrânea, caracterizada por ser rica em cereais não-refinados, frutas, vegetais e com elevada proporção de gorduras monoinsaturadas em relação às saturadas ${ }^{9,30,38}$. Segundo Vessby et al..$^{39}$ e Summers et al. ${ }^{40}$, a sensibilidade à insulina é significativamente melhorada com dietas ricas em gorduras mono ou poliinsaturadas, em comparação àquelas ricas em saturadas. Ryan et al. ${ }^{38}$ avaliaram 11 diabéticos tipo 2, cuja dieta, rica em ácido graxo linoléico, foi substituída por uma alimentação rica em ácido graxo oléico por dois meses. Tal modificação reduziu a resistência insulínica e melhorou a vasodilatação dependente do endotélio, evidenciando os benefícios antiaterogênicos da dieta mediterrânea, embora nesse estudo o número de indivíduos avaliados tenha sido pequeno. Segundo Pitsavos et al. ${ }^{9}$, a dieta mediterrânea parece atenuar o risco cardiovascular de indivíduos portadores de síndrome metabólica.

Carboidratos: de acordo com Riccardi \& Rivellese ${ }^{30}$, dietas hiperglicídicas estão associadas ao aumento das concentrações plasmáticas de 
triglicerídeos e PAI-1, bem como à redução de HDL-c e menor fibrinólise. Tais malefícios são minimizados se a digestão e absorção dos carboidratos for lenta, despertando, assim, o interesse a respeito do índice glicêmico (IG) dos alimentos. Segundo os autores, tais efeitos somente ocorrem quando alimentos de alto IG são consumidos. O IG mensura a resposta glicêmica prandial causada por um determinado alimento ${ }^{41}$. Vários fatores influenciam essa resposta, incluindo a quantidade de carboidratos, o tipo de açúcar (glicose, frutose, sacarose, lactose), a natureza do amido (amilose, amilopectina, amido resistente), o processo de cocção (grau de gelatinização do amido), a forma física do alimento e o tamanho da partícula do carboidrato ${ }^{13}$. Dietas de alto IG diminuem a sensibilidade à insulina, além de estarem associadas à hipertrigliceridemia e diminuição das concentrações plasmáticas de HDL-C $\mathrm{C}^{42}$. Por outro lado, dietas com baixos índice e carga glicêmica estão associadas a um menor risco de desenvolvimento de diabetes mellitus tipo 2, doenças cardiovasculares e certos tipos de câncer ${ }^{43}$. A carga glicêmica (CG), definida como o produto do IG por seu conteúdo de carboidratos, mensura tanto a quantidade quanto a qualidade do carboidrato consumido ${ }^{41}$.

Em estudo realizado em um hospital universitário da cidade do Rio de Janeiro, foram avaliados O IG e a CG de dietas habitualmente ingeridas por 49 pacientes diabéticos tipo 2, sem tratamento nutricional anterior. Foram utilizados, para estimar a ingestão dietética, história alimentar e questionário de freqüência de consumo de alimentos. Observou-se um alto percentual de indivíduos com excesso de peso e obesidade abdominal, dislipidemia e controle glicêmico inadequado. A maioria das refeições era de baixa $C G$, embora de alto IG, talvez devido à baixa freqüência de consumo diário de leguminosas, hortaliças e frutas ${ }^{44}$.

Visando a avaliar se dietas de CG elevada afetam as concentrações de PCR, Liu et al. ${ }^{24}$ analisaram 244 mulheres saudáveis de meia-idade, mensurando a concentração plasmática de PCR e determinando, por meio de questionário de freqüência alimentar semi-quantitativo validado, a CG média das dietas consumidas. Após análise estatística (com ajuste para idade, tabagismo, IMC, nível de atividade física, história familiar de infarto do miocárdio, história de hipertensão arterial sistêmica, diabetes mellitus e hipercolesterolemia, terapia de reposição hormonal, consumo de álcool e outras variáveis), foi encontrada forte correlação positiva entre a CG das dietas e as concentrações de $P C R$, sendo tal associação significativamente modificada pelo IMC. No entanto, mais estudos são necessários a fim de avaliar se os resultados encontrados são válidos para pacientes diabéticos, como também para portadores de síndrome metabólica.

Muitos alimentos com baixo IG são ricos em fibras alimentares, em especial as fibras solúveis ${ }^{35}$. Dados epidemiológicos recentes indicam que dietas ricas em fibras associam-se a um menor risco de doenças cardiovasculares e diabetes mellitus tipo $2^{41}$. Além disso, sabe-se que as fibras alimentares melhoram a resposta glicêmica e as concentrações de insulina prandial ${ }^{45}$.

As fibras solúveis reduzem o tempo de trânsito intestinal e ajudam na diminuição das concentrações séricas de colestero| ${ }^{34,36}$. Além disso, melhoram a tolerância à glicose, sendo responsáveis pela maioria dos benefícios cardiovasculares atribuídos às fibras alimentares ${ }^{34}$. Já as fibras insolúveis não têm ação na colesterolemia, mas aumentam a saciedade, auxiliando na redução da ingestão energética ${ }^{34,36}$. Dessa maneira, o aumento da ingestão de fibras alimentares pode promover a perda de peso ${ }^{35}$.

A recomendação de ingestão diária de fibras alimentares é de 20 a 30g, sendo que a dieta deve conter alimentos que ofereçam ao menos 5 a $10 \mathrm{~g}$ de fibras solúveis ao dia ${ }^{5}$.

Recentemente, um estudo sobre a relação entre carboidratos, resistência insulínica e prevalência de síndrome metabólica, realizado com 2834 indivíduos acometidos com essa síndrome, segundo os critérios do NCEP5, detectou que a ingestão de grãos integrais foi inversamente 
associada ao método HOMA, enquanto que o IG e a CG das dietas apresentaram correlação positiva ${ }^{46}$.

Outras pesquisas indicam que as concentrações de insulina em jejum são menores em indivíduos que relatam maior ingestão de fibras alimentares ou grãos integrais ${ }^{47,48}$. Além disso, o maior consumo de grãos integrais resulta em maior sensibilidade à ação insulínica ${ }^{49}$.

Proteínas: o aporte protéico assemelha-se ao da população geral, com ênfase no consumo de proteínas de origem vegetal (como a soja: $1,3 \mathrm{~g} \%$ de gordura saturada e $0 \mathrm{mg} \%$ de colesterol) e peixe (teor médio de $2,9 \mathrm{~g} \%$ de gordura saturada e 68mg\% de colesterol), visando à menor ingestão de gorduras saturadas e colesterol, uma vez que as carnes vermelhas apresentam teores mais elevados (teor médio de $7,3 \mathrm{~g} \%$ de gordura saturada e $85 \mathrm{mg} \%$ de colesterol $)^{5,30,50}$. A substituição de proteína de origem animal por proteína de soja diminui as concentrações sanguíneas de colesterol total e LDL-c em indivíduos hipercolesterolêmicos ${ }^{5,34}$. As concentrações séricas de LDL-c podem ser reduzidas em até $5 \%$ com dietas pobres em gorduras saturadas e colesterol, contendo $25 \mathrm{~g}$ diárias de proteína de soja ${ }^{5}$.

Em resumo, quanto ao consumo de proteínas, há um consenso de que o mesmo corresponda a $15 \%$ do $V E T^{5,30}$. No que se refere aos demais macronutrientes, os carboidratos entre $45 \%$ e $55 \%$ e as gorduras entre $30 \%$ e $40 \%{ }^{30}$. Segundo o NCEP 5 , a ingestão de carboidratos deve ser de $50 \%$ a $60 \%$ do VET e a de lipídeos de $25 \%$ a $35 \%$, sendo este último constituído por, no máximo, $10 \%$ de gorduras poliinsaturadas, $20 \%$ de monoinsaturadas e até $7 \%$ de saturadas.

Outros constituintes dietéticos: Deve-se limitar o consumo de sal, que pode prejudicar a sensibilidade à insulina ${ }^{30}$. O excesso de sódio, além de elevar a pressão arterial, aumenta a calciúria. Mesmo indivíduos normotensos podem reduzir o risco de desenvolver hipertensão arterial sistêmica consumindo menor quantidade de sal5,34,35.

O consumo de álcool deve ser também desencorajado por estar associado ao aumento das concentrações plasmáticas de triglicerídeos, quando a ingestão excede $30 \mathrm{~g}$ diárias, além de contribuir para maiores níveis pressóricos 13,30,34.

Pereira et al. ${ }^{1}$ encontraram associação inversa entre a freqüência de consumo de laticínios e o desenvolvimento de obesidade, anormalidades na homeostase de glicose, níveis pressóricos elevados e dislipidemia em homens e mulheres jovens com sobrepeso. Cálcio, potássio e magnésio parecem diminuir o risco de hipertensão arterial sistêmica, doença arterial coronariana e diabetes mellitus tipo $2^{1}$.

O magnésio participa de várias reações críticas no metabolismo de carboidratos e, por isso, acredita-se que tenha um papel importante na homeostase de glicose e ação insulínica ${ }^{51}$. Baixas concentrações de magnésio estão associadas à diminuição da sensibilidade à insulina, síndrome metabólica e diabetes mellitus tipo $2^{46}$. A fim de investigar se a ingestão de magnésio (derivada da alimentação e de suplementos) relaciona-se com o risco de desenvolver diabetes mellitus tipo 2, foram avaliadas 39345 mulheres americanas com mais de 45 anos, que participaram do Women's Health Study. Foi utilizado o questionário de freqüência alimentar semi-quantitativo, com acompanhamento por seis anos. Segundo Song et al. ${ }^{51}$, a elevada ingestão de magnésio reduz 0 risco de desenvolver diabetes mellitus tipo 2 , especialmente em mulheres com excesso de peso. Tais autores afirmaram que esses resultados foram semelhantes aos encontrados em estudos epidemiológicos americanos, como o Nurses' Health Study, The lowa Women's Health Study e Health Professionals Follow-up Study. Song et al. ${ }^{51}$ também analisaram a concentração de insulina de jejum de 349 mulheres, tendo sido encontrada associação inversa entre a ingestão de magnésio e as concentrações plasmáticas de insulina de jejum, sendo essa associação modificada pelo IMC. Tais resultados evidenciam a importância de consumir frutas, vegetais verde-escuros, grãos integrais e amêndoas, principais fontes de magnésio ${ }^{51}$.

Outro fator de destaque na fisiopatologia da síndrome metabólica é o estresse oxidativo. 
Há evidências de que a maior produção de radicais livres está inversamente correlacionada à ação insulínica ${ }^{52}$. Ford et al. ${ }^{52}$ avaliaram as concentrações plasmáticas de antioxidantes de 8808 adultos americanos com mais de 20 anos de idade, cujos dados foram obtidos do NHANES III, comparando os portadores de síndrome metabólica com aqueles que não a apresentavam. Foram avaliadas as concentrações de vitaminas $A, C$ e $E$, ésteres de retinil, cinco tipos de carotenóides e selênio. Os autores observaram que os portadores de síndrome metabólica apresentavam concentrações sub-ótimas de alguns antioxidantes, o que pode contribuir para o maior risco de desenvolvimento de diabetes mellitus e doença cardiovascular. Dessa forma, portadores de síndrome metabólica devem ser estimulados a consumir frutas e vegetais, já que são fontes de antioxidantes.

\section{O N CLUS Ã O}

A dieta desejável para portadores de síndrome metabólica deve priorizar o consumo de alimentos com baixo teor de gordura saturada e ácidos graxos trans isômeros, estimulando a ingestão de alimentos de baixo índice glicêmico e com quantidades adequadas de fibras alimentares. Deve-se, ainda, limitar o consumo de sódio. Além disso, é necessário o estímulo à prática regular de atividades físicas, com o intuito de evitar o ganho ponderal. Frente a isso, intervenções eficazes no hábito alimentar desses indivíduos são necessárias, tornando a educação nutricional um fator indispensável na prevenção e controle dessa síndrome.

\section{REFER Ê NCIAS}

1. Pereira MA, Jacobs DRJ, van Horn L, Slattery ML, Kartashov Al, Ludwig DS. Dairy consumption, obesity, and the insulin resistance syndrome in young adults: the CARDIA Study. JAMA. 2002; 287(16):2081-9.

2. Isomaa B, Almgren $P$, Tuomi T, Forsén B, Lahti $K$, Nissén $M$, et al. Cardiovascular morbidity and mortality associated with the metabolic syndrome. Diabetes Care. 2001; 24(4):683-8.

3. Grundy SM. Metabolic Syndrome: what is it and how should I treat it? ACC Curr J Rev. 2003; 12(3):37-40.

4. World Health Organization. Definition, diagnosis and classification of diabetes mellitus and its complications. Geneva; 1999. 59p.

5. Expert Panel on Detection, Evaluation and Treatment of High Blood Cholesterol in Adults (Adult Treatment Panel III). Executive Summary of the Third Report of the National Cholesterol Education Program (NCEP). JAMA. 2001; 285(19):2486-97.

6. Lorenzo C, Okoloise M, Williams K, Stern MP, Haffner SM. The metabolic syndrome as predictor of type 2 diabetes: the San Antonio heart study. Diabetes Care. 2003; 26(11):3153-9.

7. Ford ES, Giles WH. A comparison of the prevalence of the metabolic syndrome using two proposed definitions. Diabetes Care. 2003; 26(3):575-81.

8. Ford ES, Giles WH, Dietz WH. Prevalence of the metabolic syndrome among US adults - findings from the Third National Health and Nutrition Examination Survey. JAMA. 2002; 287(3):356-9.

9. Pitsavos C, Panagiotakos D, Chrysohoou C, Papaioannou I, Papadimitriou L, Tousoulis D, et al. The Adoption of Mediterranean diet attenuates the development of acute coronary syndromes in people with the metabolic syndrome. Nutr J. 2003; 2(1):1-7.

10. Santos CR, Portella ES, Avila SS, Soares EA. Identificação da síndrome metabólica em diabéticos tipo dois atendidos em Hospital Universitário do Rio de Janeiro. Rev Soc Cardiol Estado de São Paulo. 2003; 13 (2 Edição Especial):98.

11. Wallace TM, Matthews DR. The assessment of insulin resistance in man. Diabetic Med. 2002; 19(7):527-34

12. Granberry MC, Fonseca VA. Insulin resistance syndrome: options for treatment. South Med J. 1999; 92(1):2-15.

13. American Diabetes Association. Clinical practice recommendations. Diabetes Care. 2004; 27(1):S1S143.

14. Desouza C, Gilling L, Fonseca V. Management of the insulin resistance syndrome. Curr Diab Rep. 2001; 1(2):140-7.

15. Reaven G. Metabolic syndrome: pathophysiology and implications for management of cardiovascular disease. Circulation. 2002; 106(3):286-8. 
16. Barroso SG, Abreu VG, Francischetti EA. A participação do tecido adiposo visceral na gênese da hipertensão e doença cardiovascular aterogênica: um conceito emergente. Arq Bras Cardiol. 2002; 78(6):618-30.

17. Grundy SM, Brewer Jr HB, Cleeman JI, Smith SC Jr, Lenfant $C$. Definition of metabolic syndrome: report of the national heart, lung, and blood institute/ American Heart Association Conference on Scientific issues related to definition. Circulation. 2004; 109(3):433-8.

18. Grundy SM. Hypertriglyceridemia, insulin resistance and the metabolic syndrome. Am J Cardiol. 1999; 83(9B):25F-9F.

19. Souza L, Chalita F, Reis A, Teixeira C, Neto C, Bastos $D$, et al. Prevalência de diabetes mellitus e fatores de risco em Campos dos Goytacazes, RJ. Arq Bras Endocrinol Metab. 2003; 47(1):69-74.

20. Lerário DDG, Gimeno SG, Franco LJ, lunes $M$, Ferreira SRG. Excesso de peso e gordura abdominal para a síndrome metabólica em nipo-brasileiros. Rev Saúde Pública. 2002; 36(1):4-11.

21. Reseland J, Anderssen S, Solvoll K, Hjermann I, Urdal P, Holme I, et al. Effect of long-term changes in diet and exercise on plasma leptin concentrations. Am J Clin Nutr. 2001; 73(2): 240-5.

22. Fernández-Real JM, Broch M, Vendrell J, Ricart W. Insulin resistance, inflammation, and serum fatty acid composition. Diabetes Care. 2003; 26(5):1362-8.

23. Han TS, Sattar N, Williams K, Gonzalez-Villalpando C, Lean MEJ, Haffner SM. Prospective study of $C$-reactive protein in relation to the development of diabetes and metabolic syndrome in the Mexico City Diabetes Study. Diabetes Care. 2002; 25(11):2016-21.

24. Liu S, Manson J, Buring J, Stampfer M, Willett W, Ridker P. Relation between a diet with a high glycemic load and plasma concentrations of highsensitivity C-reactive protein in middle-aged women. Am J Clin Nutr. 2002; 75(3):492-8.

25. Mozaffarian D, Pischon T, Hankinson S, Rifai N, Joshipura K, Willett W, et al. Dietary intake of trans fatty acids and systemic inflammation in women. Am J Clin Nutr. 2004; 79(4):606-12.

26. Meigs J, Jacques P, Selhub J, Singer D, Nathan D, Rifai N, et al. Fasting plasma homocysteine levels in the insulin resistance syndrome: the framingham offspring study. Diabetes Care. 2001; 24(8): 1403-10

27. Godsland IF, Rosankiewicz JR, Proudler AJ, Johnston DG. Plasma total homocysteine concentrations are unrelated to insulin sensitivity and components of the metabolic syndrome in healthy men. J Clin Endocrinol Metab. 2001; 86(2):719-23.

28. Hamdy O, Ledbury S, Mullooly C, Jarema C, Porter $S$, Ovalle $K$, et al. Lifestyle modification improves endothelial function in obese subjects with the insulin resistance syndrome. Diabetes Care. 2003; 26(7):2119-25.

29. Marckmann P. Dietary treatment of thrombogenic disorders related to the metabolic syndrome. $\mathrm{Br} \mathrm{J}$ Nutr 2000; 83(Suppl 1):S121-S6.

30. Riccardi G, Rivellese AA. Dietary treatment of the metabolic syndrome: the optimal diet. $\mathrm{Br} J$ Nutr. 2000; 83(Suppl 1):S143-S8.

31. Nestel P. Nutritional aspects in the causation and management of the metabolic syndrome. Endocrinol Metab Clin North Am. 2004; 33(3):483-92.

32. Haber EP, Curi R, Carvalho CRO, Carpinelli AR. Secreção da insulina: efeito autócrino da insulina e modulação por ácidos graxos. Arq Bras Endocrinol Metab. 2001; 45(3):219-27.

33. El-Assaad W, Buteau J, Peyot M, Nolan C, Roduit $R$, Harby $S$, et al. Saturated fatty acids synergize with elevated glucose to cause pancreatic beta-cell death. Endocrinology. 2003; 144(9):4154-63.

34. Rique ABR, Soares EA, Meirelles CM. Nutrição e exercício na prevenção e controle das doenças cardiovasculares. Rev Bras Med Esp. 2002; 8(6):1-11

35. World Health Organization. Diet, nutrition and the prevention of chronic diseases. Geneva; 2003. $149 p$.

36. Sociedade Brasileira de Cardiologia. III Diretrizes Brasileiras sobre Dislipidemias e Diretriz de Prevenção da Aterosclerose do Departamento de Aterosclerose da Sociedade Brasileira de Cardiologia. Arq Bras Cardiol. 2001; 77(Suppl 3): 1-48.

37. Samaha F, Iqbal N, Seshadri P, Chicano K, Daily D, McGrory J, et al. A low carbohydrate as compared with a low fat diet in severe obesity. N Engl J Med. 2003; 348(21):2074-81.

38. Ryan M, McInerney D, Owens D, Collins P, Johnson A, Tomkin GH. Diabetes and the Mediterranean diet: a beneficial effect of oleic acid on insulin sensitivity, adipocyte glucose transport and endothelium-dependent vasoreactivity. QJM. 2000; 93(2): 85-91.

39. Vessby B, Unsitupa M, Hermansen K, Riccardi G, Rivellese A, Tapsell L, et al. Substituting dietary saturated for monounsaturated fat impairs insulin sensitivity in healthy men and women: the KANWU study. Diabetologia. 2001; 44(3):312-9. 
40. Summers L, Fielding B, Bradshaw $H$, llic V, Beysen C, Clark M, et al. Substituting dietary saturated fat with polyunsaturated fat changes abdominal fat distribution and improves insulin sensitivity. Diabetologia. 2002; 45(3):369-77.

41. Foster-Powell K, Holt SH, Brand-Miller JC. International table of glycemic index and glycemic load values: 2002. Am J Clin Nutr. 2002; 76(1):5-56.

42. Frost G, Leeds A, Dore C, Madeiros S, Brading S, Dornhorst A. Glycaemic index as a determinant of serum HDL-cholesterol concentration. Lancet. 1999; 353(9158):1045-8.

43. Brand-Miller J, Hayne S, Petocz P, Colagiuri S. Lowglycemic index diets in the management of diabetes: a meta-analysis of randomized controlled trials. Diabetes Care. 2003; 26(8):2261-7.

44. Santos CRB, Portella ES, Avila SS, Soares EA. Avaliação do índice e CG de dietas ingeridas por diabéticos tipo 2. In: Libro de memorias del XIII Congreso Latinoamericano de Nutrición; 2003. Acapulco, México. México: Argiletum Editores; 2003. p.223-4.

45. Meyer K, Kushi L, Jacobs D Jr, Slavin J, Sellers T, Folsom A. Carbohydrates, dietary fiber, and incident type 2 diabetes in older women. Am J Clin Nutr. 2000; 71(4):921-30.

46. McKeown NM, Meigs JB, Liu S, Saltzman E, Wilson PWF, Jacques PF. Carbohydrate nutrition, insulin resistance, and the prevalence of the metabolic syndrome in the Framingham Offspring Cohort. Diabetes Care. 2004; 27(2):538-46.
47. Ludwig D, Pereira M, Kroenke C, Hilner J, van Horn $L$, Slattery $M$, et al. Dietary fiber, weight gain, and cardiovascular disease risk factors in young adults. JAMA. 1999; 282(16):1539-46.

48. McKeown NM, Meigs JB, Liu S, Wilson PW, Jacques PF. Whole-grain intake is favorably associated with metabolic risk factors for type 2 diabetes and cardiovascular disease in the Framingham Offspring Study. Am J Clin Nutr. 2002; 76(2): 390-8.

49. Pereira M, Jacobs D Jr, Pins J, Raatz S, Gross M, Slavin J, et al. Effect of whole grains on insulin sensitivity in overweight hyperinsulinemic adults. Am J Clin Nutr. 2002; 75(5):848-55.

50. Philippi ST. Tabela de composição de alimentos: suporte para decisão nutricional. São Paulo: Coronário; 2002. 107p.

51. Song Y, Manson JE, Buring JE, Liu S. Dietary magnesium intake in relation to plasma insulin levels and risk of type 2 diabetes in women. Diabetes Care. 2004; 27(1):59-65.

52. Ford ES, Mokdad AH, Giles WH, Brown DW. The metabolic syndrome and antioxidant concentrations: findings from the Third National Health and Nutrition Examination Survey. Diabetes. 2003; 52(9):2346-52.

Recebido em: 29/1/2004

Versão final reapresentada em: 5/1/2005

Aprovado em: 9/3/2005 